\title{
eTUTOR: ONLINE LEARNING FOR PERSONALIZED EDUCATION
}

\author{
Cem Tekin \\ Bilkent University, Ankara, Turkey
}

\begin{abstract}
Given recent advances in information technology and artificial intelligence, web-based education systems have became complementary and, in some cases, viable alternatives to traditional classroom teaching. The popularity of these systems stems from their ability to make education available to a large demographics (see MOOCs). However, existing systems do not take advantage of the personalization which becomes possible when web-based education is offered: they continue to be one-size-fits-all. In this paper, we aim to provide a first systematic method for designing a personalized web-based education system. Personalizing education is challenging: (i) students need to be provided personalized teaching and training depending on their contexts (e.g. classes already taken, methods of learning preferred, etc.), (ii) for each specific context, the best teaching and training method (e.g type and order of teaching materials to be shown) must be learned, (iii) teaching and training should be adapted online, based on the scores/feedback (e.g. tests, quizzes, final exam, likes/dislikes etc.) of the students. Our personalized online system, eTutor, is able to address these challenges by learning how to adapt the teaching methodology (in this case what sequence of teaching material to present to a student) to maximize her performance in the final exam, while minimizing the time spent by the students to learn the course (and possibly dropouts). We illustrate the efficiency of the proposed method on a real-world eTutor platform which is used for remedial training for a Digital Signal Processing (DSP) course.
\end{abstract}

Index Terms - Online learning, personalized education, eLearning, intelligent tutoring systems.

\section{INTRODUCTION}

The last decade has witnessed an explosion in the number of web-based education systems due to the increasing demand in higher-level education [1], limited number of teaching personnel, and advances in information technology and artificial intelligence. Nowadays, most universities have integrated Massive Open Online Course (MOOC) platforms into their education systems such as edX consortium, Coursera or Udacity [2-4], to give students the possibility to learn by interacting with a software program instead of human teachers. Several advantages of these systems over traditional classroom teaching are: (i) they provide flexibility to the student in choosing what to learn and when to learn, (ii) they do not require the presence of an interactive human teacher, (iii) there

\author{
Jonas Braun, Mihaela van der Schaar
}

\author{
University of California, Los Angeles, CA
}

are no limitations in terms of the number of students who can take the course. However, there are significant limitations of currently available online teaching platforms. Since courses are taken online, there is no interaction between the students and the teacher as in a classroom setting. This makes it very difficult to meet the personalized needs of each student, which may arise due to the differences between qualifications, learning methods and cognitive skills of the students. It is observed that if the personalization of teaching content is not carried out efficiently, high drop-outs will occur [1]. For instance, the students that are very familiar with the topic may dropout if the teaching material is not challenging enough, while the students that are new to the topic may get overstrained if the teaching material is hard.

Due to these challenges, a new web-based education system that personalizes education by learning online the needs of the students based on their contexts, and adapting the teaching material based on the feedback signals received from the student (answers to questions, quizzes, etc.) is required. For this purpose we develop the eTutor (illustrated in Fig. 1), which is an online web-based education system, that learns how to teach a course, a concept or remedial materials to a student with a specific context in the most efficient way. Basically, for the current student, eTutor learns from its past interactions with students with similar contexts, the sequence of teaching materials that are shown to these students, and the response of these students to the teaching materials including the final exam scores, how to teach the course in the most effective way. This is done by defining a teaching effectiveness metric, referred to as the regret, that is a function of the final exam score and time cost of teaching to the student, and then designing a learning algorithm that learns to optimize this metric. This tradeoff between learning (exploring) and optimizing (exploiting) is captured by the eTutor in the most efficient way, i.e., the average exam score of the students converge to the average exam score that could be achieved by the best teaching strategy. We illustrate the efficiency of the proposed system in a real-world experiment carried out on students in a DSP class.

\subsection{Related Work}

Although web-based education systems have recently become popular, there is no consensus or standards on how to design an optimal web-based education system. A detailed comparison of our work with the related work in web-based education 


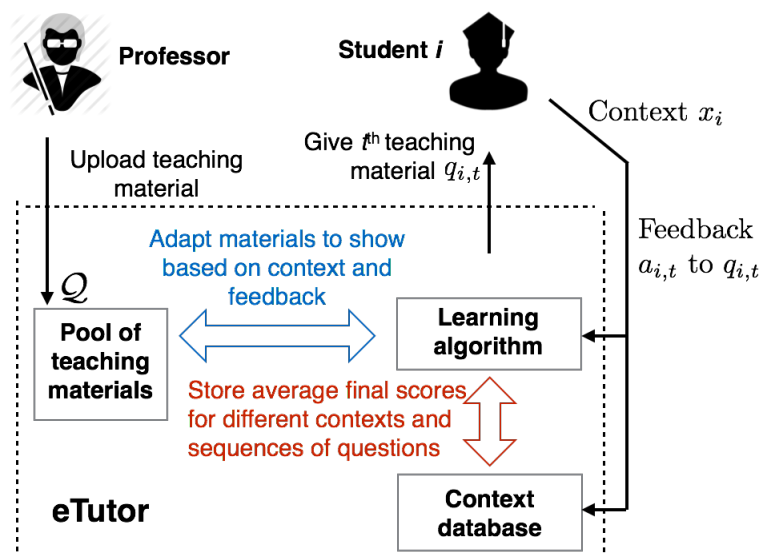

Fig. 1. eTutor, student and professor interaction.

\begin{tabular}{|l|c|c|c|c|}
\hline Method & $\begin{array}{c}\text { Context-based } \\
\text { learning }\end{array}$ & $\begin{array}{c}\text { Feedback-based } \\
\text { learning }\end{array}$ & $\begin{array}{c}\text { Learns from } \\
\text { final exam }\end{array}$ & $\begin{array}{c}\text { Regret } \\
\text { bound }\end{array}$ \\
\hline$[5,6]$ & Yes & No & No & No \\
\hline$[7-13]$ & No & Yes & No & No \\
\hline Our work & Yes & Yes & Yes & Yes \\
\hline
\end{tabular}

Table 1. Comparison with related work.

is given in Table 1. Most of the recent works focus on the subfield of MOOCs, which are online courses with very large number of students [1-4]. Among these, several works examine students' interaction with commercially available MOOC systems such as Coursera [2,3] and edX [4].

Apart from these, two approaches exist in designing webbased education systems: adaptive education systems and intelligent tutoring systems. In an adaptive education system $[5,6]$, the teaching materials that are shown to each student are adapted based on the context of the student, but not based on the feedback the student provides during the course. This adaptation is based on numerous contexts including the student's learning style, her knowledge, background, origin, grades, previously taken courses etc. In contrast, in an intelligent tutoring system adaptation is done based on the response of the student to the given teaching material [7-13], without taking into account contexts. Our work combines both ideas by adapting the sequence of teaching materials that is presented to a student based on both the context and the feedback of the student. However, our techniques are very different from both lines of research. Our goal is to learn the optimal way to teach a course in a way that is most effective for each student. To learn effectively, our method utilizes the past knowledge gained about the efficacy of the material from students with similar contexts who have taken the course before. This is different from [7-13], which only take into account the current student's response to the previously shown teaching material.

\section{FORMALISM, ALGORITHM AND ANALYSIS}

In this section we mathematically formalize the online teaching/tutoring problem, define a benchmark tutor (i.e. the "ideal" tutor) and propose an online learning algorithm for the eTutor which converges in performance to the benchmark tutor that knows the optimal sequence of teaching materials to show for each student.

\subsection{Problem Definition}

Consider a set of students participating in an online education system and a concept that should be learned by the students. The comprehension of the concept will be tested via a final exam (test). We assume that the students arrive sequentially over time and use index $i$ to denote the $i$ th student. Additionally, we assume that when a student first interacts with the online education system, she needs to answer a set of questions, which will form the context of the student. Context may include information about the student such as age, grades, whether she prefers visual or written instructions, etc. Denote the finite set of all possible contexts by $\mathcal{X}$ and an element of $\mathcal{X}$ by $x$. The concept will be taught by presenting a set of teaching materials (written or visual) to the student and asking a set of questions about these materials and providing their answers. Let $\mathcal{Q}$ be the set of teaching materials (consists of text/images to learn from and questions) that can be given to the student. The number of elements of $\mathcal{Q}$ is denoted by $Q$.

The materials that are shown to a student are chosen in an online way based on the context of the student, previous materials that are shown to the student, the student's response to shown questions (whether the answer is correct or not) and all the previous knowledge obtained from past students with contexts, responses and scores similar to the current student. It is also important to learn in which order the materials should be shown, since learning from one material may require knowledge of a concept which can be learned by understanding another material.

For each student $i$, we consider a discrete time model $t=1,2, \ldots, T_{i}$, where time $t$ denotes the sequence of events related to the $t$ th material that is shown to the student. $T_{i}$ denotes the number of teaching materials shown to student $i$ before the final exam is given (depends on student's feedback). Clearly, $T_{i} \leq Q$. The $t$ th teaching material shown to student $i$ is denoted by $q_{i, t}$. Let $\boldsymbol{q}_{i}:=\left(q_{i, 1}, \ldots, q_{i, T_{i}}\right)$, and $\boldsymbol{q}_{i}[t]:=\left(q_{i, 1}, \ldots, q_{i, t}\right)$.

We denote student $i$ 's response to $q_{i, t}$ by $a_{i, t} \in\{-1,0,1\}$. If the student does not provide any feedback on the teaching material, we have $a_{i, t}=0$; when the teaching material is a (multiple-choice) question, $a_{i, t}=1$ denotes a correct answer and $a_{i, t}=-1$ denotes a wrong answer. Let $\boldsymbol{a}_{i}:=\left(a_{i, 1}, \ldots, a_{i, T_{i}}\right)$ and $\boldsymbol{a}_{i}[t]:=\left(a_{i, 1}, \ldots, a_{i, t}\right), t \leq T_{i}$. In addition, let $a_{i, 0}:=0$, which indicates that no feedback is available prior to 1st teaching material. Although we consider the specific feedback model given above, our algorithm and analysis can easily be generalized to any feedback model in which a student's feedback to every teaching material comes from a finite set.

Let $\boldsymbol{S}$ denote the set of all sequences of teaching materials that can be shown. ${ }^{1}$ For a sequence of materials $s \in S$, let $\mathcal{A}(s)$ be the set of sequences of feedbacks a student can pro-

\footnotetext{
${ }^{1}$ In practice, it is possible to give $\boldsymbol{S}$ as an input in addition to $\mathcal{Q}$. For instance, some sequences which are classified by the professor as unreasonable can be discarded, significantly reducing the size of $\boldsymbol{S}$.
} 
vide. The expected final exam score for a student with context $x$, sequence of questions $\boldsymbol{s} \in \boldsymbol{S}$ and sequence of feedbacks $\boldsymbol{a} \in \mathcal{A}(\boldsymbol{s})$ is denoted by $r_{x, s, \boldsymbol{a}}$. We assume that the final exam score of a student with context $x$, the sequence of teaching materials $s$ and the sequence of feedbacks $\boldsymbol{a}$ is randomly drawn from a $F_{x, s, a}$ with expected value $r_{x, s, a}$. Both $F_{x, \boldsymbol{s}, \boldsymbol{a}}$ and $r_{x, \boldsymbol{s}, \boldsymbol{a}}$ are unknown.

\subsection{The Benchmark Tutor}

Due to the enormous number of possible sequences of teaching materials, it is not possible to learn the best sequence of teaching materials by trying all of them for different students. In this section we define a benchmark tutor, whose teaching strategy can be learned very fast. We call it the best-first (BF) benchmark. Due to limited space its pseudocode is given in our online appendix [14], however, we describe it in detail below. In order to explain this benchmark, we require a few more notations.

Given a sequence $s$ of teaching materials, let $\mathcal{Q}_{s}$ be the set of remaining teaching materials that can be given to the student. Let $\boldsymbol{S}[t] \subset \boldsymbol{S}$ be the set of sequences that consists of $t$ teaching materials followed by the final exam. In order to explicitly state the number of teaching materials in a sequence of teaching materials, we will use the notation $s[t]$ to denote an element of $\boldsymbol{S}[t]$. We will also use $\boldsymbol{a}_{\boldsymbol{s}[t]}\left(t^{\prime}\right)$ to denote the student's feedback to the first $t^{\prime}$ teaching materials in $\boldsymbol{s}[t]$. Let $y_{x, \boldsymbol{s}[t], \boldsymbol{a}_{\boldsymbol{s}[t]}(t-1)}=E_{a_{t}}\left[r_{x, \boldsymbol{s}[t],\left(\boldsymbol{a}_{\boldsymbol{s}[t]}(t-1), a_{t}\right)}\right]$ be the ex-ante final exam score of a student with context $x$ which is given teaching materials $s[t]$ and provided feedback to all of them except the last teaching material.

The BF benchmark incrementally selects the next teaching material to show based on the student's feedback about the previous teaching materials. The first teaching material it shows is $q_{x, 1}^{*}=\arg \max _{q \in \mathcal{Q}} y_{x, q, 0}$. Let $q_{x, t}^{*}$ be the $t$ th teaching material that this benchmark shows, which depends on $\boldsymbol{a}^{*}[t-1]$. Let $\boldsymbol{q}_{x}^{*}$ be the sequence of teaching materials shown by the BF benchmark. We have $q_{x, t}^{*}=$ $\arg \max _{q \in \mathcal{Q}_{\boldsymbol{q}_{x}^{*}[t-1]}} r_{x, \boldsymbol{q}_{x}^{*}[t-1], \boldsymbol{a}_{t-1}^{*}}$. For any $t$, if $r_{x, \boldsymbol{q}_{x}^{*}[t], \boldsymbol{a}^{*}[t]} \geq$ $y_{x,\left(\boldsymbol{q}_{x}^{*}[t], q\right), \boldsymbol{a}^{*}[t]}-c$ for all $q \in \mathcal{Q}\left(\boldsymbol{q}_{x}^{*}[t]\right)$, then the BF benchmark will give the final exam after the $t$ th teaching material. Here $c>0$ is the teaching cost of showing one more material to the student, which is the cost related to the time it takes for the student to complete the teaching material. The average final exam score minus the teaching cost achieved by following the $\mathrm{BF}$ benchmark for the first $n$ students is equal to $R W_{\mathrm{BF}}(n)=\sum_{i=1}^{n} E\left[Y_{x_{i}, \boldsymbol{Q}_{i}^{*}, \boldsymbol{A}_{i}^{*}}-c\left|\boldsymbol{Q}_{i}^{*},\right|\right] / n$, where $Y_{x_{i}, \boldsymbol{Q}_{i}^{*}, \boldsymbol{A}_{i}^{*}}$ is the random variable that represents the final exam score of student $i$, where $\boldsymbol{Q}_{i}^{*}$ is the random variable that represents the sequence of teaching materials given to student $i$ by the BF benchmark, and $\boldsymbol{A}_{i}^{*}$ is the random variable that represents the sequence of feedbacks provided by student $i$ to the teaching materials $\boldsymbol{Q}_{i}^{*}$. The BF benchmark is an $o r$ acle policy because we assume that nothing is known about the expected exam scores a priori. Any learning algorithm $\alpha$ which selects a sequence of teaching materials $\boldsymbol{Q}_{i}^{\alpha}$ based

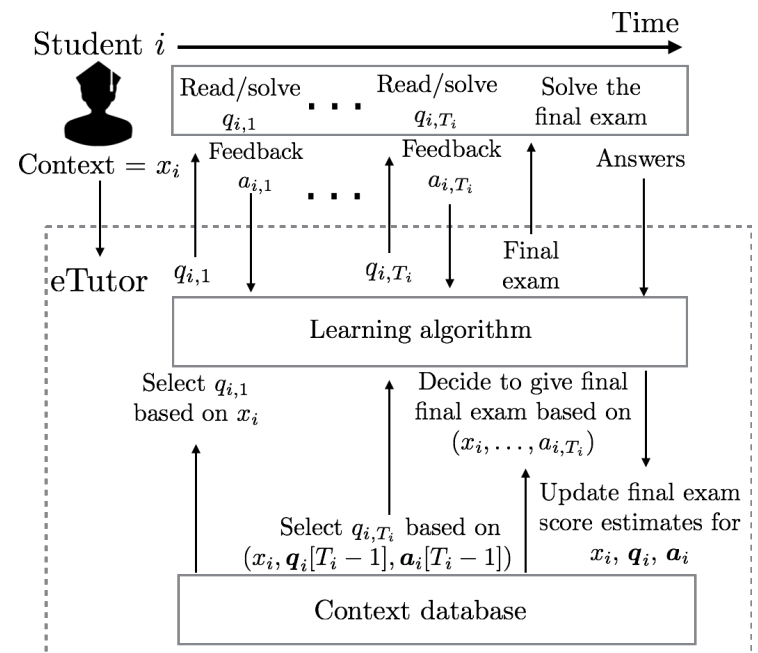

Fig. 2. Operation of the eTutor.

on the sequence of feedbacks $\boldsymbol{A}_{i}^{\alpha}$ has a average regret with respect to the $\mathrm{BF}$ benchmark which is given by

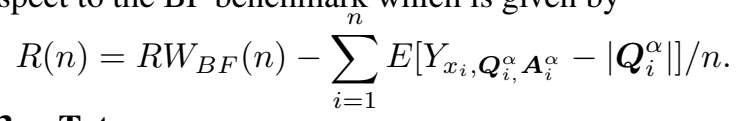

\section{3. eTutor}

In this section we propose eTutor (pseudocode given in our online appendix [14] due to limited space), which learns the optimal sequence of teaching materials to show based on the student's context and feedback about the previously shown teaching materials (as shown in Fig. 2). In order to minimize the regret given in (1), eTutor balances exploration and exploitation when selecting the teaching materials to show to the student. Consider a student $i$ and the $t$ th teaching material shown to that student. eTutor keeps the following sample mean reward estimates: (i) $\hat{r}_{x, t, q, a}(i)$ which is the estimated final exam score for students with context $x$ that took the course before student $i$ who are given the final exam right after material $q$ is given as the $t$ th material and feedback $a$ is observed, (ii) $\hat{y}_{x, a, t, q}(i)$ which is the estimated final exam score for students with context $x$ that took the course before student $i$ who are given the final exam right after material $q$ is given as the $t$ th material after observing feedback $a$ for the $t-1$ th material. In addition to these, eTutor keeps the following counters: (i) $T_{x, t, q, a}(i)$ which counts the number of times material $q$ is shown as the $t$ th material and feedback $a$ is obtained for students with context $x$ that took the course before student $i$, (ii) $T_{x, a, t, q}(i)$ which counts the number of times material $q$ is shown as the $t$ th material after feedback $a$ is obtained from the previously shown material for students with context $x$ that took the course before student $i$.

Next, we explain how exploration and exploitation is performed. Consider the event that eTutor asks question $q_{i, t}=q$ and receives feedback $a_{i, t}=a$. It first checks if $T_{x, t, q, a}(i)<$ $D \log i$, where $D>0$ is a constant that is an input parameter of eTutor. If this holds, then eTutor explores by giving the final exam and obtaining the final score $X(i)$, by which it updates $\hat{r}_{x, t, q, a}(i+1)=\left(\hat{r}_{x, t, q, a}(i+1)+X(i)\right) /\left(T_{x, t, q, a}(i)+1\right)$. Else if $T_{x, t, q, a}(i) \geq D \log i$, eTutor checks if there are any 
questions $q^{\prime} \in \mathcal{Q}_{\boldsymbol{q}_{i}[t]}$ for which $T_{x, a_{i, t}, t+1, q^{\prime}}(i)<D \log i$. If there are such questions, then eTutor explores one of them randomly by showing that material to the student, obtaining the feedback, giving the final exam, and obtaining the final exam score. The obtained final exam score $X(i)$ is used for both updating $\hat{r}_{x, t+1, q^{\prime}, a_{i, t+1}}(i+1)$ and $\hat{y}_{x, a_{i, t}, t+1, q^{\prime}}(i+1)$. If none of the above events happen, then eTutor exploits at $t$. To do this it first checks if $\hat{r}_{x, t, q, a_{i, t}}(i) \geq \hat{y}_{x, a_{i, t}, t+1, q^{\prime}}(i)-c$, for all $q^{\prime} \in \mathcal{Q}_{\boldsymbol{q}_{i}[t]}$. If this is the case, it means that showing one more teaching material does not increase the final exam score enough to compensate for the teaching cost of showing one more material. Hence, eTutor gives the final exam after its $t$ th material. If the opposite happens, then it means that showing one more material can improve final exam score sufficiently enough for it to compensate the cost of teaching. Hence, eTutor will show one more teaching material to the student which is $q_{i, t+1}=\arg \max _{q^{\prime} \in \mathcal{Q}_{\boldsymbol{q}_{i}[t]}} \hat{y}_{x, a_{i, t}, t+1, q^{\prime}}(i)$. The next decision to take will be based on the student's feedback to $q_{i, t+1}$ which is $a_{i, t+1}$. This goes on until eTutor gives the final exam, which will eventually happen since $\mathcal{Q}$ is finite.

\subsection{Regret bound for eTutor}

Given that the constant $D$ that is input to eTutor is such that $D \geq 4 / \Delta_{\text {min }}^{2}$, where $\Delta_{\min }$ is the minimum over all optimal sequence of teaching materials corresponding to different feedbacks, the minimum difference between the final exam score of that optimal sequence of teaching materials and a suboptimal sequence of teaching materials. We have the following bound on the regret.

Theorem 1 The regret of eTutor for the first $n$ students is bounded as

$$
R(n)=O(|\mathcal{X}| Q D \log n / n) .
$$

Proof: (Sketch) We can write $R(n)=R_{e}(n)+R_{s}(n)$, where $R_{e}(n)$ is the regret due to explorations and $R_{s}(n)$ is the regret due to suboptimal material selections at exploitations. The bound on $R_{e}(n)$ comes from the fact that for each $i=1,2, \ldots, n$ and for each tuple $(\boldsymbol{s}, \boldsymbol{a})$, $s \in \mathcal{S}, a \in \mathcal{A}(s)$ and $x \in \mathcal{X}$, eTutor only exploits the best estimated sequence of teaching materials after at least $D \log i$ final exam score observations are made. Due to this, the order of explorations is $O(\log n)$. The bound on $R_{s}(n)$ comes from the fact that when $D \geq$ $4 / \Delta_{\min }^{2}$, for any $i \in\{1, \ldots, n\}$ for which eTutor exploits, $\mathrm{P}\left(\left|\hat{r}_{x_{i}, t, q_{i, t}, a_{i, t}}(i)-r_{x_{i}, \boldsymbol{q}_{i}[t], \boldsymbol{a}_{i}[t]}\right| \geq \Delta_{\min } / 2\right)=O\left(i^{-2}\right)$ and $\mathrm{P}\left(\left|\hat{y}_{x_{i}, a_{i, t}, t+1, q^{\prime}}(i)-y_{x_{i},\left(\boldsymbol{q}_{i}[t], q^{\prime}\right), \boldsymbol{a}_{i}[t]}\right| \geq \Delta_{\min } / 2\right)=O\left(i^{-2}\right)$

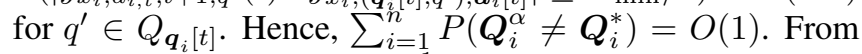
this, we have $R_{s}(n)=O\left(n^{-1}\right)$.

Theorem 1 implies that the average final exam score of students tutored by eTutor converges to the average final exam score of students tutored by BL which knows the expected final exam scores, and hence, how students learn for each sequence of teaching materials perfectly. Moreover, the regret gives the convergence rate, and since it decreases with $\log n / n$, eTutor converges very fast.

\begin{tabular}{|l|c|c|}
\hline \# of students & $n=100$ & $n=500$ \\
\hline eTutor & $(66.4,8.7)$ & $(75.8,8.5)$ \\
\hline RR & $(62.4,10.2)$ & $(62.5,10.2)$ \\
\hline FR & $(75.5,17.0)$ & $(75.0,17.0)$ \\
\hline
\end{tabular}

Table 2. Comparison of eTutor with RR and FR: For each entry $(x, y), x$ denotes the average final score $($ maximum $=100)$ and $y$ denotes the time spent in minutes taking the course.

\section{ILLUSTRATIVE RESULTS}

We deployed our eTutor system for students who have already studied digital signal processing (DSP) one or more years ago, and the goal of this implementation of the eTutor is to have them refresh the material about discrete Fourier transform (DFT) in the minimum amount of time. Student contexts belong to $\mathcal{X}=\{0,1\}$, where for a student $i, x_{i}=0$ implies that she is not confident about her knowledge of DFT, and $x_{i}=1$ implies that she is confident about her knowledge of DFT. $\mathcal{Q}$ contains three (remedial) materials: one text that describes DFT and two questions that refreshes DFT knowledge. If a question is shown to the student and if the student's answer is incorrect, then the correct answer is shown along with an explanation. For each $q \in \mathcal{Q}$, we set the cost to be $c_{q}=0.04 \times \theta_{q}$, where $\theta_{q}$ (in minutes) is the average time it takes for a student to complete material $q$. The value of $\theta_{q}$ is estimated and updated based on the responses of the students. Performance of the students after taking the remedial materials are tested by the same final exam.

We compare the performance of eTutor with a random rule (RR) that randomly selects the materials to show and a fixed rule (FR) that shows all materials (text first, easy question second, hard question third). The average final score achieved by these algorithms for $n=100$ and $n=500$ students are shown in Table 2. From this table we see that eTutor achieves $15,7 \%$ and $1.1 \%$ improvement in the average final score for $n=500$ compared to RR and FR, respectively. The improvement compared to FR is small because FR shows all the materials to every student. It is observed that the average final score of eTutor increases with $n$, which is expected since eTutor learns the best set of materials to show for each context as more students take the course. In contrast, RR and FR are non-adaptive, hence their average final exam scores do not improve as more students take the course. For $n=500$, the average time spent by each student taking the course is 8.5 minutes for eTutor which is $16.7 \%$ and $50 \%$ less than the average time it takes for the same set of students by RR and FR, respectively. eTutor achieves significant savings in time by showing the best materials to each student based on her context instead of showing everything to every student.

\section{CONCLUSION}

In this paper, we proposed a novel online education system called eTutor. While in this paper, eTutor was used to learn the best sequence of materials to show to a specific student, eTutor can also be easily adapted to learn the best teaching methodology such as what types of materials/examples to show (visual or not), what style of teaching to use etc. 


\section{REFERENCES}

[1] C. Brinton and M. Chiang, "Social learning networks: A brief survey," in Proc. of the 48th Annual Conference on Information Sciences and Systems (CISS), March 2014, pp. 1-6.

[2] A. Anderson, D. Huttenlocher, J. Kleinberg, and J. Leskovec, "Engaging with massive online courses," in Proc. of the 23rd International World Wide Web Conference, 2014.

[3] R. F. Kizilcec, C. Piech, and E. Schneider, "Deconstructing disengagement: analyzing learner subpopulations in massive open online courses," in Proc. of the 3rd Conference on Learning Analytics and Knowledge, 2013, pp. 170-179.

[4] L. Breslow, D. E. Pritchard, J. DeBoer, G. S. Stump, A. D. Ho, and D. T. Seaton, "Studying learning in the worldwide classroom research into edX's first MOOC," Research and Practice in Assessment, pp. 13-25, 2013.

[5] S. Guven, "Mltutor: a web-based educational adaptive hypertext system," in Lost in the Web - Navigation on the Internet (Ref. No. 1999/169), IEE Colloquium, 1999, pp. $4 / 1-4 / 3$.

[6] N. Henze and W. Nejdl, "Adaptation in open corpus hypermedia," International Journal of Artificial Intelligence in Education, pp. 325-350, 2001.

[7] A. Mitrovic, "An intelligent sql tutor on the web," International Journal of Artificial Intelligence in Education, pp. 171-195, 2003.

[8] T. Heift and D. Nicholson, "Web delivery of adaptive and interactive language tutoring," International Journal of Artificial Intelligence in Education, pp. 310-324, 2001.

[9] K. Forbes-Riley, D. Litman, and M. Rotaru, "Responding to student uncertainty during computer tutoring: A preliminary evaluation," in Proc. of the 9th International Conference on Intelligent Tutoring Systems (ITS), 2008.

[10] S. Schiaffino, P. Garcia, and A. Amandi, "eteacher: Providing personalized assistance to e-learning students," Computers and Education, vol. 51, no. 4, pp. 1744 1754, 2008.

[11] A. Zouhair, E.-M. En-Naimi, B. Amami, H. Boukachour, P. Person, and C. Bertelle, "Intelligent tutoring systems founded of incremental dynamic case based reasoning and multi-agent systems (its-idcbr-mas)," in Proc. of the 2013 International Conference on Advanced Logistics and Transport (ICALT), May 2013, pp. 341346.
[12] S. Piramuthu, "Knowledge-based web-enabled agents and intelligent tutoring systems," IEEE Transactions on Education, vol. 48, no. 4, pp. 750-756, Nov 2005.

[13] S. Nafiseh and M. Ali, "Evaluation based on personalization using optimized firt and mas framework in engineering education in e-learning environment," in Proc. of the 4th International Conference on E-Learning and E-Teaching (ICELET), Feb 2013, pp. 117-120.

[14] C. Tekin, J. Braun, and M. van der Schaar, "Online appendix for: etutor: Online learning for personalized education," http://medianetlab.ee.ucla.edu/papers/etutor. 